PROCEEDINGS OF THE

AMERICAN MATHEMATICAL SOCIETY

Volume 125, Number 8, August 1997, Pages 2275-2283

S $0002-9939(97) 04148-8$

\title{
THE EXISTENCE OF POSITIVE SOLUTIONS FOR THE ONE-DIMENSIONAL $p$-LAPLACIAN
}

\author{
JUNYU WANG
}

(Communicated by Hal L. Smith)

\begin{abstract}
In this paper we study the existence of positive solutions of the equation $\left(g\left(u^{\prime}\right)\right)^{\prime}+a(t) f(u)=0$, where $g(v)=|v|^{p-2} v, p>1$, subject to nonlinear boundary conditions. We show the existence of at least one positive solution by a simple application of a Fixed Point Theorem in cones and the Arzela-Ascoli Theorem.
\end{abstract}

\section{INTRODUCTION}

In a recent paper [2], Erbe and Wang considered the boundary value problem

$$
\begin{cases}u^{\prime \prime}+a(t) f(u)=0, & 0<t<1, \\ \alpha u(0)-\beta u^{\prime}(0)=0, & \gamma u(1)+\delta u^{\prime}(1)=0,\end{cases}
$$

under the following three assumptions:

$$
\begin{gathered}
\alpha, \beta, \gamma, \delta \geq 0 \text { and } \rho:=\alpha \gamma+\alpha \delta+\beta \gamma>0 . \\
f \in C([0,+\infty),[0,+\infty)) .
\end{gathered}
$$

$$
a \in C([0,1],[0,+\infty)) \text { and } a(t) \not \equiv 0 \text { on any subinterval of }[0,1] .
$$

They obtained the following existence result:

Theorem 1. Assume (A1)-(A3) hold. Then the boundary value problem (1.1) has at least one positive solution in the case

(i) $f_{0}=0$ and $f_{\infty}=+\infty$, or

(ii) $f_{0}=+\infty$ and $f_{\infty}=0$,

where

$$
f_{0}:=\lim _{u \downarrow 0} \frac{f(u)}{u}, \quad f_{\infty}:=\lim _{u \uparrow+\infty} \frac{f(u)}{u} .
$$

The proof of Theorem 1 is based on the following Fixed Point Theorem due to Krasnoselskii [3].

Received by the editors December 6, 1995

1991 Mathematics Subject Classification. Primary 34B15.

Key words and phrases. One-dimensional p-Laplacian, positive solution, existence, concavity, fixed point theorem in cones.

The author was supported by NNSF of China. 
Theorem 2 ([1], [3]). Let $E$ be a Banach space, and let $K \subset E$ be a cone in $E$. Assume $\Omega_{1}, \Omega_{2}$ are open subsets of $E$ with $0 \in \Omega_{1}, \bar{\Omega}_{1} \subset \Omega_{2}$, and let

$$
\Phi: K \cap\left(\bar{\Omega}_{2} \backslash \Omega_{1}\right) \rightarrow K
$$

be a completely continuous operator such that either

(i) $\|\Phi u\| \leq\|u\| \forall u \in K \cap \partial \Omega_{1}$, and $\|\Phi u\| \geq\|u\| \forall u \in K \cap \partial \Omega_{2}$; or

(ii) $\|\Phi u\| \geq\|u\| \forall u \in K \cap \partial \Omega_{1}$, and $\|\Phi u\| \leq\|u\| \forall u \in K \cap \partial \Omega_{2}$.

Then $\Phi$ has a fixed point in $K \cap\left(\bar{\Omega}_{2} \backslash \Omega_{1}\right)$.

In another recent paper [4], Yang and Fan investigated the boundary value problem

$$
\left\{\begin{array}{l}
\left(g\left(u^{\prime}\right)\right)^{\prime}+u^{a}=0, \quad 0<t<1, a \in(0, p-1) \cup(p-1,+\infty), \\
u(0)=0, \quad u^{\prime}(1)=0
\end{array}\right.
$$

where $g(v):=|v|^{p-2} v, p>1$, and demonstrated that the problem (1.2) has at least one positive solution, by applying Theorem 2 .

Motivated by the results mentioned above, in this paper we study the existence of positive solutions of the quasilinear differential equation

$$
\left(g\left(u^{\prime}\right)\right)^{\prime}+a(t) f(u)=0, \quad 0<t<1,
$$

subject to one of the following three pairs of boundary conditions:

$$
\begin{gathered}
u(0)-B_{0}\left(u^{\prime}(0)\right)=0, \quad u(1)+B_{1}\left(u^{\prime}(1)\right)=0, \\
u(0)-B_{0}\left(u^{\prime}(0)\right)=0, \quad u^{\prime}(1)=0, \\
u^{\prime}(0)=0, \quad u(1)+B_{1}\left(u^{\prime}(1)\right)=0,
\end{gathered}
$$

where $g(v):=|v|^{p-2} v, p>1$, and hence $\left(g\left(u^{\prime}\right)\right)^{\prime}$ is the one-dimensional $p$-Laplacian.

The following hypotheses are adopted throughout this paper:

(H1) $B_{0}(v)$ and $B_{1}(v)$ are both nondecreasing, continuous, odd functions defined on $(-\infty,+\infty)$ and at least one of them satisfies the condition that there exists $b>0$ such that

$$
0 \leq B_{j}(v) \leq b v \quad \text { for all } v \geq 0, j=1 \text { or } 2 .
$$

(H2) $f(u)$ is a nonnegative, lower semi-continuous function defined on $[0,+\infty)$. Moreover, it has only a finite number of discontinuity points of the first kind in each compact subinterval of $[0,+\infty)$.

(H3) $a(t)$ is a nonnegative measurable function defined on $(0,1)$ and satisfies the following conditions:

$$
0<\int_{0}^{1 / 2} G\left(\int_{s}^{1 / 2} a(t) d t\right) d s+\int_{1 / 2}^{1} G\left(\int_{1 / 2}^{s} a(t) d t\right) d s<+\infty,
$$

if $B_{0}(v) \equiv 0$ and $B_{1}(v) \equiv 0$;

$$
0<\int_{0}^{1} G\left(\int_{s}^{1} a(t) d t\right) d s<+\infty
$$

if $B_{0}(v) \equiv 0$ and $B_{1}(v) \not \equiv 0\left(\right.$ or $\left.u^{\prime}(1)=0\right)$;

$$
0<\int_{0}^{1} G\left(\int_{0}^{s} a(t) d t\right) d s<+\infty
$$


if $B_{1}(v) \equiv 0$ and $B_{0}(v) \not \equiv 0$ (or $\left.u^{\prime}(1)=0\right)$; and

$$
0<\int_{0}^{1} a(t) d t<+\infty
$$

if $B_{0}(v) \not \equiv 0$ (or $u^{\prime}(0)=0$ ) and $B_{1}(v) \equiv 0$ (or $\left.u^{\prime}(0)=0\right)$, where $G(w)=$ $|w|^{1 /(p-1)} \operatorname{sgn} w$ is the inverse function to $g(v)$.

When $p=2,\left(1.6_{\mathrm{a}}\right),\left(1.6_{\mathrm{b}}\right)$, and $\left(1.6_{\mathrm{c}}\right)$ can respectively be written as

$$
\begin{aligned}
& 0<\int_{0}^{1 / 2} t a(t) d t+\int_{1 / 2}^{1}(1-t) a(t) d t<+\infty \\
& 0<\int_{0}^{1} t a(t) d t<+\infty \\
& 0<\int_{0}^{1}(1-t) a(t)<+\infty
\end{aligned}
$$

which more clearly show that $a(t)$ is allowed to have singularity at the endpoints of $(0,1)$. In addition, (H3) allows $a(t) \equiv 0$ on some subintervals of $(0,1)$. For example, the function

$$
a(t)= \begin{cases}t^{-\alpha}, & 0<t<1 / 8 ; 1<\alpha<p, \\ 0, & 1 / 8 \leq t<1,\end{cases}
$$

satisfies $(\mathrm{H} 3)$ provided $B_{0}(v) \equiv 0$.

It is obvious that (1.1) and (1.2) are both particular cases of the boundary value problem (1.3)-(1.4). The aim of this paper is to extend and improve the existence results in [2], [4].

By a positive solution of the boundary value problem (1.3)-(1.4), we mean a function $u(t)$ satisfying the following conditions:

(i) $u \in C[0,1] \cap C^{1}(0,1)$ if $B_{0}(v) \equiv 0$ and $B_{1}(v) \equiv 0$;

$u \in C[0,1] \cap C^{1}(0,1]$, if $B_{0}(v) \equiv 0$ and $B_{1}(v) \not \equiv 0$ (or $\left.u^{\prime}(1)=0\right)$;

$u \in C[0,1] \cap C^{1}[0,1)$, if $B_{1}(v) \equiv 0$ and $B_{0}(v) \not \equiv 0$ (or $\left.u^{\prime}(0)=0\right)$;

$u \in C^{1}[0,1]$, if $B_{0}(v) \not \equiv 0$ (or $\left.u^{\prime}(0)=0\right)$ and $B_{1}(v) \not \equiv 0\left(\right.$ or $\left.u^{\prime}(1)=0\right)$.

(ii) $u(t)>0$ for all $t \in(0,1)$ and satisfies boundary conditions in (1.3).

(iii) $g\left(u^{\prime}(t)\right)$ is locally absolutely continuous in $(0,1)$ and the equality

$$
\left[g\left(u^{\prime}(t)\right)\right]^{\prime}=-a(t) f(u(t))
$$

holds almost everywhere in $(0,1)$.

It is clear that $u \equiv 0$ is a solution of (1.3)-(1.4) when $f(0)=0$ and each solution of (1.3)-(1.4) is a nonnegative concave function defined in $[0,1]$. However, the concavity of solutions has not been employed in both [2] and [4].

The main result of this paper is as follows.

Theorem 3. Assume (H1)-(H3) hold. Then the boundary value problem (1.3)(1.4) has at least one positive solution in the case

(i) $f_{0}=0$ and $f_{\infty}=+\infty$, or

(ii) $f_{0}=+\infty$ and $f_{\infty}=0$;

where

$$
f_{0}:=\lim _{u \downarrow 0} \frac{f(u)}{u^{p-1}}, \quad f_{\infty}:=\lim _{u \uparrow+\infty} \frac{f(u)}{u^{p-1}}
$$


Clearly, Theorem 3 is an extension and improvement of the existence results in [2], [4].

Our arguments involve the use of the concavity and integral representation of solutions, Theorem 2, and the Arzela-Ascoli Theorem.

\section{Proof of Theorem 3}

It follows from (1.6) that there exists $\delta \in(0,1 / 2)$ such that

$$
0<\int_{\delta}^{1-\delta} a(t) d t<+\infty
$$

and hence the function

$$
y(x):=\int_{\delta}^{x} G\left(\int_{s}^{x} a(t) d t\right) d s+\int_{x}^{1-\delta} G\left(\int_{x}^{s} a(t) d t\right) d s, \quad \delta \leq x \leq 1-\delta,
$$

is continuous and positive on $[\delta, 1-\delta]$. In the sequel $\delta$ always satisfies (2.1).

Let $K$ be the cone in $C[0,1]$ given by

$$
K:=\{u \in C[0,1] ; u(t) \text { is a nonnegative concave function }\} .
$$

Lemma 1. Let $u \in K$ and $\delta \in(0,1 / 2)$. Then

$$
\begin{aligned}
& u(t) \geq\left\{\begin{array}{ll}
\|u\| t / \sigma, & 0 \leq t \leq \sigma, \\
\|u\|(1-t) /(1-\sigma), & \sigma \leq t \leq 1,
\end{array} \text { if } 0<\sigma<1,\right. \\
& u(t) \geq\|u\| t, \quad 0 \leq t \leq 1, \text { if } \sigma=1, \\
& u(t) \geq\|u\|(1-t), \quad 0 \leq t \leq 1, \text { if } \sigma=0,
\end{aligned}
$$

Here $\|u\|:=\sup \{|u(t)| ; 0 \leq t \leq 1\}$ and $\sigma \in[0,1]$ such that $u(\sigma)=\|u\|$.

Proof. The lemma follows from the concavity of $u(t)$ on $[0,1]$.

Now we define an operator $\Phi: K \rightarrow K$ by

$$
W(t)=(\Phi u)(t):=\left\{\begin{array}{c}
B_{0} \circ G\left(\int_{0}^{\sigma} a(r) f(u(r)) d r\right)+\int_{0}^{t} G\left(\int_{s}^{\sigma} a(r) f(u(r)) d r\right) d s, \\
0 \leq t \leq \sigma, \\
B_{1} \circ G\left(\int_{\sigma}^{1} a(r) f(u(r)) d r\right)+\int_{t}^{1} G\left(\int_{\sigma}^{s} a(r) f(u(r)) d r\right) d s, \\
\quad \sigma \leq t \leq 1,
\end{array}\right.
$$


for each $u \in K$, where $\sigma=0$ if $W^{\prime}(0)=0 ; \sigma=1$ if $W^{\prime}(1)=0$; otherwise, $\sigma$ is a solution of the equation

$$
\begin{aligned}
z_{0}(x)= & z_{1}(x), \\
z_{0}(x):= & B_{0} \circ G\left(\int_{0}^{x} a(r) f(u(r)) d r\right) \\
& +\int_{0}^{x} G\left(\int_{s}^{x} a(r) f(u(r)) d r\right) d s, \quad 0 \leq x<1, \\
z_{1}(x):= & B_{1} \circ G\left(\int_{x}^{1} a(r) f(u(r)) d r\right) \\
& +\int_{x}^{1} G\left(\int_{x}^{s} a(r) f(u(r)) d r\right) d s, \quad 0<x \leq 1 .
\end{aligned}
$$

The equation (2.4) has at least one solution in $(0,1)$, because $z_{0}(x)$ is a nondecreasing continuous function defined on $[0,1)$ with $z_{0}(0)=0$ and $z_{1}(x)$ a nonincreasing continuous function defined on $(0,1]$ with $z_{1}(1)=0$. Moreover, if $\sigma_{1}, \sigma_{2} \in[0,1]$, $\sigma_{1}<\sigma_{2}$, are solutions of (2.4), then we have $a(r) f(u(r)) \equiv 0$ on $\left[\sigma_{1}, \sigma_{2}\right]$. Therefore, the operator $\Phi$ is well defined.

From the definition of $\Phi$, we deduce that for each $u \in K, W=\Phi u \in K$ and satisfies (1.4) and $W(\sigma)$ is the maximum value of $W$ on $[0,1]$, since

$$
W^{\prime}(t)= \begin{cases}G\left(\int_{t}^{\sigma} a(r) f(u(r)) d r\right) \geq 0, & 0<t \leq \sigma, \\ -G\left(\int_{\sigma}^{t} a(r) f(u(r)) d r\right) \leq 0, & \sigma \leq t<1,\end{cases}
$$

is continuous and nonincreasing in $(0,1)$ and $W^{\prime}(\sigma)=0$. Moreover,

$$
\left[g\left(W^{\prime}(t)\right)\right]^{\prime}=-a(t) f(u(t)) \quad \text { a.e. in }(0,1)
$$

This shows that $\Phi(K) \subset K$ and each fixed point of $\Phi$ in $K$ is a solution of (1.3)(1.4).

Lemma 2. Assume that $f(u)$ is continuous on $[0,+\infty)$. Then (1.3)-(1.4) has a positive solution $u \in K$. Moreover, there exist two positive numbers $R_{1}, R_{2}$ such that

$$
0<R_{1} \leq\|u\| \leq R_{2} .
$$

Proof. Let $u \in K$ and $W=\Phi u$. The proof is similar to that of Theorem 1. Under our hypotheses, we need to distinguish the following eight cases:

(1) $W(0)-B_{0}\left(W^{\prime}(0)\right)=0, W(1)+B_{1}\left(W^{\prime}(1)\right)=0, B_{0}(v) \not \equiv 0, B_{1}(v) \not \equiv 0$;

(2) $W(0)=0, W(1)+B_{1}\left(W^{\prime}(1)\right)=0, B_{1}(v) \not \equiv 0$;

(3) $W(0)-B_{0}\left(W^{\prime}(0)\right)=0, W(1)=0, B_{0}(v) \not \equiv 0$;

(4) $W(0)=0, W(1)=0$

(5) $W(0)-B_{0}\left(W^{\prime}(0)\right)=0, W^{\prime}(1)=0, B_{0}(v) \not \equiv 0$;

(6) $W(0)=0, W^{\prime}(1)=0$;

(7) $W^{\prime}(0)=0, W(1)+B_{1}\left(W^{\prime}(1)\right)=0, B_{1}(v) \not \equiv 0$; and

(8) $W^{\prime}(0)=0, W(1)=0$.

First we deal with case (1). In this case, it is easy to see that $\Phi: K \rightarrow K$ is completely continuous.

Now suppose that $f_{0}=0$ and $f_{\infty}=+\infty$. Without loss of generality, we may assume that

$$
0 \leq B_{0}(v) \leq b v \text { for all } v \geq 0
$$


Since $f_{0}=0$, we choose $R_{1}>0$ so that

$$
0 \leq f(u) \leq(\varepsilon u)^{p-1} \quad \text { whenever } 0 \leq u \leq R_{1},
$$

where $\varepsilon>0$ satisfies

$$
\varepsilon(b+1) G\left(\int_{0}^{1} a(r) d r\right)<1 .
$$

Thus, if $u \in K$ and $\|u\|=R_{1}$, then from (2.5) and (2.6)

$$
\begin{aligned}
\|W\|=W(\sigma) & \leq B_{0} \circ G\left(\int_{0}^{1} a(r) f(u(r)) d r\right)+G\left(\int_{0}^{1} a(r) f(u(r)) d r\right) \\
& \leq B_{0}\left(\varepsilon R_{1} G\left(\int_{0}^{1} a(r) d r\right)\right)+\varepsilon R_{1} G\left(\int_{0}^{1} a(r) d r\right) \\
& \leq R_{1} \varepsilon(b+1) G\left(\int_{0}^{1} a(r) d r\right)<R_{1}=\|u\| .
\end{aligned}
$$

Now, if we let $\Omega_{1}:=\left\{u \in C[0,1] ;\|u\|<R_{1}\right\}$, then (2.7) shows that

$$
\|\Phi u\|<\|u\| \quad \forall u \in K \cap \partial \Omega_{1} .
$$

Further, since $f_{\infty}=+\infty$, there exists $R_{2}>R_{1} / \delta$ such that

$$
f(u) \geq(M u)^{p-1} \quad \text { whenever } u \geq \delta R_{2},
$$

where $M>0$ is chosen so that

$$
\delta L M>2, \quad L:=\min \{y(x) ; \delta \leq x \leq 1-\delta\} .
$$

Let $\Omega_{2}=\left\{u \in C[0,1] ;\|u\|<R_{2}\right\}$. Then for $u \in K$ and $\|u\|=R_{2}$, from (2.3) and (2.10), we have

$$
\begin{aligned}
2\|W\| & \geq \int_{0}^{\sigma} G\left(\int_{s}^{\sigma} a(r) f(u(r)) d r\right) d s+\int_{\sigma}^{1} G\left(\int_{\sigma}^{s} a(r) f(u(r)) d r\right) d s \\
& \geq \int_{\delta}^{\sigma} G\left(\int_{s}^{\sigma} a(r) f(u(r)) d r\right) d s+\int_{\sigma}^{1-\delta} G\left(\int_{\sigma}^{s} a(r) f(u(r)) d r\right) d s \\
& \geq \delta M R_{2}\left[\int_{\delta}^{\sigma} G\left(\int_{s}^{\sigma} a(r) d r\right) d s+\int_{\sigma}^{1-\delta} G\left(\int_{\sigma}^{s} a(r) d r\right) d s\right] \\
& \geq \delta L M R_{2}>2 R_{2}=2\|u\|, \quad \text { if } \sigma \in[\delta, 1-\delta], \\
\|W\| & \geq \int_{\delta}^{1-\delta} G\left(\int_{\delta}^{s} a(r) f(u(r)) d r\right) d s \\
& \geq \delta L M R_{2}>2 R_{2}>\|u\|, \quad \text { if } \sigma>1-\delta, \\
\|W\| & \geq \int_{\delta}^{1-\delta} G\left(\int_{\delta}^{s} a(r) f(u(r)) d r\right) d s \\
& \geq \delta L M R_{2}>\|u\|, \quad \text { if } \sigma<\delta,
\end{aligned}
$$

i.e.,

$$
\|\Phi u\|>\|u\| \quad \forall u \in K \cap \partial \Omega_{2} .
$$

Therefore, by the first part of the Fixed Point Theorem, it follows that $\Phi$ has a fixed point $u \in K \cap\left(\bar{\Omega}_{2} \backslash \Omega_{1}\right)$. Furthermore, since $0<R_{1}<\|u\|<R_{2}$, it follows from $(2.2)$ that $u(t)>0$ for $t \in(0,1)$. This shows that the fixed point $u$ is a positive solution of (1.3)-(1.4). 
Suppose next that $f_{0}=+\infty$ and $f_{\infty}=0$. Since $f_{0}=+\infty$, we may choose $R_{1}>0$ such that

$$
f(u) \geq(M u)^{p-1} \quad \text { whenever } 0 \leq u \leq R_{1},
$$

where $M>0$ satisfies (2.10). Then for $u \in K$ and $\|u\|=R_{1}$, we have

$$
\begin{aligned}
2\|W\| & \geq \int_{\delta}^{\sigma} G\left(\int_{s}^{\sigma} a(r) f(u(r)) d r\right) d s+\int_{\sigma}^{1-\delta} G\left(\int_{\sigma}^{s} a(r) f(u(r)) d r\right) d s \\
& \geq \delta M R_{1}\left[\int_{\delta}^{\sigma} G\left(\int_{s}^{\sigma} a(r) d r\right) d s+\int_{\sigma}^{1-\delta} G\left(\int_{\sigma}^{s} a(r) d r\right) d s\right] \\
& \geq \delta L M R_{1}>2 R_{1}=2\|u\|, \quad \text { if } \sigma \in[\delta, 1-\delta], \\
\|W\| & \geq \int_{\delta}^{1-\delta} G\left(\int_{s}^{1-\delta} a(r) f(u(r)) d r\right) d s \\
& \geq \delta L M R_{1}>2 R_{1}>\|u\|, \quad \text { if } \sigma>1-\delta, \\
\|W\| & \geq \int_{\delta}^{1-\delta} G\left(\int_{\delta}^{s} a(r) f(u(r)) d r\right) d s \\
& \geq \delta L M R_{1}>\|u\|, \quad \text { if } \sigma<\delta,
\end{aligned}
$$

i.e.,

$$
\|\Phi u\|>\|u\| \quad \forall u \in K \cap \partial \Omega_{1} .
$$

Now, since $f_{\infty}=0$, there exists $R_{0}>0$ so that

$$
f(u) \leq(\varepsilon u)^{p-1} \quad \text { whenever } u \geq R_{0},
$$

where $\varepsilon>0$ satisfies (2.6).

If $f$ is unbounded, then we choose $R_{2}>R_{0}+R_{1}$ so that

$$
f(u) \leq f\left(R_{2}\right) \quad \text { for } 0 \leq u \leq R_{2} .
$$

(We are able to do this since $f$ is unbounded.) For $u \in K$ and $\|u\|=R_{2}$, we have

$$
\begin{aligned}
\|W\| & \leq B_{0} \circ G\left(\int_{0}^{1} a(r) f(u(r)) d r\right)+G\left(\int_{0}^{1} a(r) f(u(r)) d r\right) \\
& \leq B_{0} \circ G\left(f\left(R_{2}\right)\right) G\left(\int_{0}^{1} a(r) d r\right)+G\left(f\left(R_{2}\right)\right) G\left(\int_{0}^{1} a(r) d r\right) \\
& \leq(b+1) \varepsilon R_{2} G\left(\int_{0}^{1} a(r) d r\right)<R_{2}=\|u\| .
\end{aligned}
$$


If $f$ is bounded, say $f(u) \leq N^{p-1}$ for all $u \geq 0$. In this case, let $R_{2}>R_{1}+N / \varepsilon$. Then for $u \in K$ with $\|u\|=R_{2}$, we have

$$
\begin{aligned}
\|W\| & \leq B_{0} \circ G\left(\int_{0}^{1} a(r) f(u(r)) d r\right)+G\left(\int_{0}^{1} a(r) f(u(r)) d r\right) \\
& \leq B_{0} \circ\left(N G\left(\int_{0}^{1} a(r) d r\right)+N\left(\int_{0}^{1} a(r) d r\right)\right) \\
& \leq(b+1) N G\left(\int_{0}^{1} a(r) d r\right) \\
& <(b+1) \varepsilon R_{2} G\left(\int_{0}^{1} a(r) d r\right)<R_{2}=\|u\| .
\end{aligned}
$$

Therefore, in either case we may put $\Omega_{2}:=\left\{u \in C[0,1] ;\|u\|<R_{2}\right\}$, and we have

$$
\|\Phi u\|<\|u\| \quad \forall u \in K \cap \partial \Omega_{2} .
$$

By the second part of Theorem 2, it follows that (1.3)-(1.4) has a positive solution.

We can deal with the remaining possibilities in a similar way and therefore we conclude that Lemma 2 is valid.

Lemma 3. Assume that $f(u)$ is discontinuous on $[0,+\infty)$. Then the conclusion of Lemma 2 still holds.

Proof. Applying linear approximation in neighborhoods of discontinuity points of $f$, we can construct a sequence $\left\{f_{j}(u)\right\}_{j=1}^{\infty}$ satisfying the following conditions:

(i) $f_{j} \in C[0,+\infty)$ and $0 \leq f_{j}(u) \leq f_{j+1}(u)$ on $[0,+\infty), j=1,2, \ldots$,

(ii) $\lim _{j \rightarrow \infty} f_{j}(u)=f(u)$ pointwise on $[0,+\infty)$,

by (H2). Lemma 2 tells us that the boundary value problem (1.3)-(1.4) with $f=f_{j}$ has a positive solution

$$
u_{j}(t)=\left\{\begin{array}{c}
B_{0} \circ G\left(\int_{0}^{\sigma_{j}} a(r) f_{j}\left(u_{j}(r)\right) d r\right)+\int_{0}^{t} G\left(\int_{s}^{\sigma_{j}} a(r) f_{j}\left(u_{j}(r)\right) d r\right) d s, \\
0 \leq t \leq \sigma_{j}, \\
B_{1} \circ G\left(\int_{\sigma_{j}}^{1} a(r) f_{j}\left(u_{j}(r)\right) d r\right)+\int_{t}^{1} G\left(\int_{\sigma_{j}}^{s} a(r) f_{j}\left(u_{j}(r)\right) d r\right) d s, \\
\quad \sigma_{j} \leq t \leq 1,
\end{array}\right.
$$

where $\sigma_{j}$ is a maximum point of $u_{j}(t)$. Moreover, $0<R_{1} \leq\left\|u_{j}\right\| \leq R_{2}$ with $R_{1}, R_{2}$ independent of $j$. From

$$
\int_{0}^{1}\left|u_{j}^{\prime}(t)\right| d t \leq 2\left\|u_{j}\right\| \leq 2 R_{2}, \quad j=1,2, \ldots,
$$

it follows that $\left\{u_{j}(t)\right\}_{j=1}^{\infty}$ is equicontinuous on $[0,1]$. Thus the existence of uniformly convergent subsequences follows from the Arzela-Ascoli Theorem. We may without loss of generality assume that $\left\{u_{j}(t)\right\}_{j=1}^{\infty}$ converges to $u(t)$ uniformly on $[0,1], R_{1} \leq$ $\|u\| \leq R_{2}$, and $\left\{\sigma_{j}\right\}_{j=1}^{\infty}$ is a monotone (nondecreasing or nonincreasing) sequence 
which tends to $\sigma \in[0,1]$. We apply Fatou's Lemma to obtain

$$
u(t) \geq\left\{\begin{array}{c}
B_{0} \circ G\left(\int_{0}^{\sigma} a(r) \varliminf_{j \rightarrow \infty} f\left(u_{j}(r)\right) d r\right)+\int_{0}^{t} G\left(\int_{s}^{\sigma} a(r) \varliminf_{j \rightarrow \infty} f\left(u_{j}(r)\right) d r\right) d s, \\
0 \leq t \leq \sigma, \\
B_{1} \circ G\left(\int_{\sigma}^{1} a(r) \varliminf_{j \rightarrow \infty} f\left(u_{j}(r)\right) d r\right)+\int_{t}^{1} G\left(\int_{\sigma}^{s} a(r) \varliminf_{j \rightarrow \infty} f\left(u_{j}(r)\right) d r\right) d s, \\
\sigma \leq t \leq 1 .
\end{array}\right.
$$

On the other hand, we have

$$
u(t) \leq\left\{\begin{array}{c}
B_{0} \circ G\left(\int_{0}^{\sigma_{j}} a(r) f\left(u_{j}(r)\right) d r\right)+\int_{0}^{t} G\left(\int_{s}^{\sigma_{j}} a(r) f\left(u_{j}(r)\right) d r\right) d s \\
0 \leq t \leq \sigma_{j} \\
B_{1} \circ G\left(\int_{\sigma_{j}}^{1} a(r) f\left(u_{j}(r)\right) d r\right)+\int_{t}^{1} G\left(\int_{\sigma_{j}}^{s} a(r) f\left(u_{j}(r)\right) d r\right) d s \\
\sigma_{j} \leq t \leq 1
\end{array}\right.
$$

Letting $j \rightarrow \infty$ in the above, we get

$$
u(t) \leq\left\{\begin{array}{c}
B_{0} \circ G\left(\int_{0}^{\sigma} a(r) f(u(r)) d r\right)+\int_{0}^{t} G\left(\int_{s}^{\sigma} a(r) f(u(r)) d r\right) d s, \\
0 \leq t \leq \sigma, \\
B_{1} \circ G\left(\int_{\sigma}^{1} a(r) f(u(r)) d r\right)+\int_{t}^{1} G\left(\int_{\sigma}^{s} a(r) f(u(r)) d r\right) d s, \\
\sigma \leq t \leq 1 .
\end{array}\right.
$$

The inequality in (2.13) and (2.14) becomes an equality, since

$$
\varliminf_{j \rightarrow \infty} f\left(u_{j}(r)\right) \geq f(u(r)) \quad \text { for all } r \in[0,1] .
$$

Therefore, the function $u(t)$ is a positive solution of (1.3)-(1.4). This completes the proof of the lemma.

Theorem 3 is proved.

\section{REFERENCES}

1. K. Deimling, Nonlinear functional analysis, Springer, New York, 1985. MR 86j:47001

2. L. H. Erbe and H. Wang, On the existence of positive solutions of ordinary differential equations, Proc. Amer. Math. Soc. 120 (1994), 743-748. MR 94e:34025

3. M. A. Krasnoselskii, Positive solutions of operator equations, Noordhoff, Gronignen, 1964. MR 31:6107

4. Z. Yang and X. Fan, The existence of positive solutions of a class of two-order quasilinear boundary value problems, Natural Science Journal of Xiangtan University, 15 (1993), Suppl. 205-209. MR 95j:34037

Department of Mathematics, Jilin University, Changchun 130023, People's Republic OF CHINA 